\title{
Self-Sensing TDR for Bearing Failure Detection of CFRP Laminate Fastener Hole with Particular Reference to the Effect of Fasteners
}

\author{
Akira Todoroki', Keisuke Ohara², Yoshihiro Mizutani1, Yoshiro Suzuki', \\ Ryosuke Matsuzaki ${ }^{3}$ \\ ${ }^{1}$ Department of Mechanical Sciences of Engineering, Tokyo Institute of Technology, Tokyo, Japan \\ ${ }^{2}$ Tokyo Institute of Technology, Tokyo, Japan \\ ${ }^{3}$ Department of Mechanical Engineering, Tokyo University of Science, Chiba, Japan \\ Email: atodorok@ginza.mes.titech.ac.jp
}

Received 21 May 2015; accepted 8 June 2015; published 11 June 2015

Copyright (C) 2015 by authors and Scientific Research Publishing Inc.

This work is licensed under the Creative Commons Attribution International License (CC BY). http://creativecommons.org/licenses/by/4.0/

\section{(c) (i) Open Access}

\begin{abstract}
Carbon fiber reinforced polymer composites (CFRP) have been applied to aerospace and automobile structures. For many CFRP structures, mechanical metallic fasteners are usually adopted. For the fasteners used in internal structures such as a wing box, the damage to the CFRP structures around fastener holes is visually quite difficult to find. A simple method to find the damage around fastener holes is required. In this study a self-sensing time domain reflectometry (TDR) method is newly applied to detect bearing failure around the fastener holes of CFRP structures. A microstrip-line method is generally used to create a transmission line. When the transmission line is mounted near the metallic fasteners, they may affect the impedance of the transmission line. In this study, the effect of distance between the fasteners and the transmission line was numerically investigated using a finite difference time domain analysis method. After finding the appropriate distance, experiments were performed to detect the bearing failure around a fastener hole. The experiments showed the performance of the self-sensing TDR for detecting bearing failure.
\end{abstract}

\section{Keywords}

Composites, Time Domain Reflectometry, Self-Sensing, Bearing Failure, Fasteners, Monitoring

\section{Introduction}

Carbon fiber reinforced polymer composites (CFRP) have been applied to aerospace and automobile structures.

How to cite this paper: Todoroki, A., Ohara, K., Mizutani, Y., Suzuki, Y. and Matsuzaki, R. (2015) Self-Sensing TDR for Bearing Failure Detection of CFRP Laminate Fastener Hole with Particular Reference to the Effect of Fasteners. Open Journal of Composite Materials, 5, 60-69. http://dx.doi.org/10.4236/ojcm.2015.53009 
For many CFRP structures, metallic fasteners are usually adopted to connect many components. For the fasteners used in the internal structures, such as a wing box, the damage to CFRP structures around the fastener holes is quite difficult to find visually. A simple method for finding the damage around the fastener holes is required for actual CFRP structures. The typical damage around fastener holes is reported to be a bearing failure mode in the review paper of Thoppul et al. [1]. Methods for monitoring bearing failure damage around fastener holes have been published in several papers. Galea et al. [2] proposed a method using piezo film. Ihn and Chang [3] used SMART Layer consisting of a thin film with distributed piezoelectric sensors. Thostenson and Chou [4] proposed a new sensor made from carbon nano-tubes to monitor the initiation of damage around fastener holes.

Several research articles have been published on self-sensing time domain reflectometry (TDR) methods for CFRP structures. Copper tape and CFRP have been used as a couple of conductive materials, and glass fiber reinforced polymer (GFRP) as an electric insulator to create a transmission line for the electro-magnetic waves [5]-[8]. The self-sensing TDR has been applied to metallic materials [9].

Chen et al. [10] embedded a coaxial cable in a concrete structure to detect cracks in the concrete structure using the TDR method. Lin et al. [11] described a method to measure applied strain using a coaxial cable with the TDR method. Yankielum et al. [12] proposed a monitoring of a bridge using the TDR method. Obaid et al. [13] used a TDR method to measure the delamination crack length of a CFRP laminate. The TDR method is normally used to check disconnection of a transmission line such as a coaxial cable, and the equipment required for the TDR method is now reasonably priced at several hundred dollars. The TDR method enables us to monitor damage location at a low cost. The self-sensing TDR method requires only the installation of transmission lines on target structures that have electrical conductivity, such as metallic or CFRP structures.

In this study, therefore, the self-sensing TDR method is newly applied to detect bearing failure damage around the fastener holes of CFRP structures. While curved transmission lines have been applied on the CFRP structural surface [8], straight transmission lines were adopted in this study. Bearing failure around the fastener holes is monitored using a straight transmission line.

The self-sensing TDR method enabled us to detect the bearing damage to lines of fasteners that had small wiring dimensions even for internal CFRP structures. The TDR requires a couple of conductive materials separated by an insulator material that electrically insulates them. The self-sensing TDR method uses a CFRP structure and copper tape as conductive materials and GFRP as an insulator. A micro-strip line (MSL) method is generally used to create a transmission line. When the transmission line is mounted near the metallic fasteners, they may affect the characteristic impedance of the transmission line, which may cause ghost damage or a scatter of electro-magnetic waves.

In this study, the effect of the distance between the fasteners and transmission line was numerically investigated using a finite difference time domain (FDTD) analysis method. After finding an appropriate distance, experimental investigation was performed to detect the bearing failure around a fastener hole, and the detection of a multiple damage situation was also investigated experimentally.

\section{FDTD Analysis}

In this study, FDTD analysis was adopted to investigate the effect of distance between the electric-conductive fastener and the transmission line. The FDTD analysis numerically solves the well-known Maxwell's equations. Because the TDR method uses a transmission line for the electro-magnetic waves, solving the Maxwell's equations numerically gives simulated results of the self-sensing TDR method. CFRP can be treated as an electrically conductive material with orthotropic conductivity as shown in Kurokawa et al. [6] which also shows the computational method. The difference is the boundary condition. In this study, the absorbing boundary condition proposed by Mur [14] was used to reduce the computational cost sacrificing long term stability. The computational reliability was confirmed by comparing the previous and new results using Mur's absorbing boundary condition.

A MSL type transmission line was used in this study as shown in Figure 1: a narrow copper strip tape and a CFRP laminate were used as the two conducting materials and a GFRP plate as an insulator between them. The characteristic impedance of the narrow-strip transmission line was obtained as shown by Wadell [15].

$$
Z_{c}=\frac{Z_{c}^{a}}{\sqrt{\varepsilon_{W}}}
$$




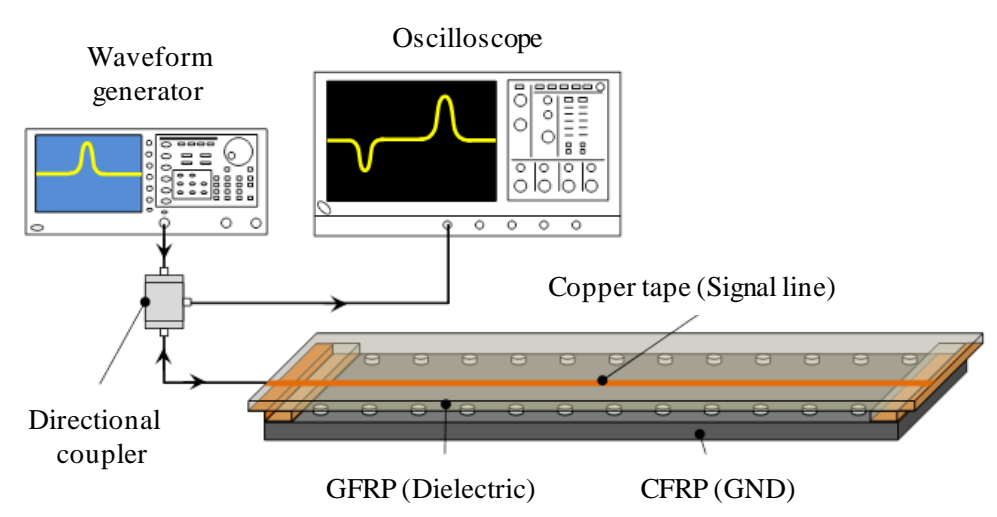

Figure 1. Schematic representation of the self-sensing TDR method.

$$
\begin{aligned}
& Z_{c}^{a}=30 \ln \left[1+\frac{4 h}{W_{0}}\left\{\frac{8 h}{W_{0}}+\sqrt{\left(\frac{8 h}{W_{0}}\right)^{2}+\pi^{2}}\right\}\right] \\
& \varepsilon_{W}=\frac{\varepsilon_{r}+1}{2}+\frac{\varepsilon_{r}-1}{2}\left(1+\frac{10 h}{W}\right)^{\frac{1}{2}}-\frac{\varepsilon_{r}-1}{4.6} \frac{t}{h} \sqrt{\frac{h}{W}} \\
& W_{0}=W+\frac{t}{\pi} \ln \frac{4 \mathrm{e}}{\left[\left(\frac{t}{h}\right)^{2}+\frac{1}{\pi^{2}\left(\frac{W}{t}+1.1\right)^{2}}\right]^{\frac{1}{2}}}
\end{aligned}
$$

where $h$ is the height of the dielectric GFRP, $\varepsilon_{\mathrm{r}}$ the relative permittivity of the GFRP, $W$ the width of the copper strip, $t$ its thickness, $Z_{c}^{a}$ the characteristic impedance when the dielectric material is a vacuum and $\varepsilon_{W}$ the effective value of relative permittivity. Using Equations (1) to (4), the characteristic impedance of the narrow-strip transmission line can be designed to match the impedance of a coaxial cable of $50 \Omega$. Without impedance matching, the input pulse signal is entirely reflected at the input end of the specimen transmission line. Impedance matching is an indispensable procedure for detecting damage in CFRP structures using the self-sensing TDR method.

Because the thickness of GFRP and copper tape is fixed at a low value, the impedance matching to the coaxial cable of $50 \Omega$ means the dimensions of the MSL are almost confined to narrow bands. In this study the dimension was fixed to a MSL $10 \mathrm{~mm}$ wide and $0.4 \mathrm{~mm}$ thick, and the insulator was 1-mm-thick GFRP.

As normal fasteners are made from metallic material, the existence of fasteners near the MSL affects the electromagnetic wave propagation because of the electromagnetic wave energy emitted around the MSL. Most of the energy is, however, concentrated between the copper tape strip and CFRP plate. This means a small part of the electromagnetic wave energy is reflected at the metallic fasteners when they exist close to the MSL.

The analysis model is shown in Figure 2. The CFRP plate is $940 \mathrm{~mm}$ long, $120 \mathrm{~mm}$ wide and $2 \mathrm{~mm}$ thick. A GFRP plate $1 \mathrm{~mm}$ thick is mounted on the surface of the CFRP plate specimen and 10-mm wide copper tape is attached to the GFRP plate near the fastener line. As shown in Figure 2, the coordinate $x$ defines the direction along the length of the CFRP specimen, $y$ across its width and the $\mathrm{z}$ the direction along its depth. The dimensions of the unit cell for the FDTD analysis are $(\Delta x, \Delta y, \Delta z)=(1 \mathrm{~mm}, 1 \mathrm{~mm}, 0.2 \mathrm{~mm})$. The analysis dimensions are $1024 \mathrm{~mm}$ long by $136 \mathrm{~mm}$ wide by $64 \mathrm{~mm}$ deep including the surrounding boundary area. The time step for the FDTD analysis should satisfy Courant's stability condition as follows:

$$
\Delta t \leq \frac{1}{c \sqrt{\frac{1}{\Delta x^{2}}+\frac{1}{\Delta y^{2}}+\frac{1}{\Delta z^{2}}}}
$$




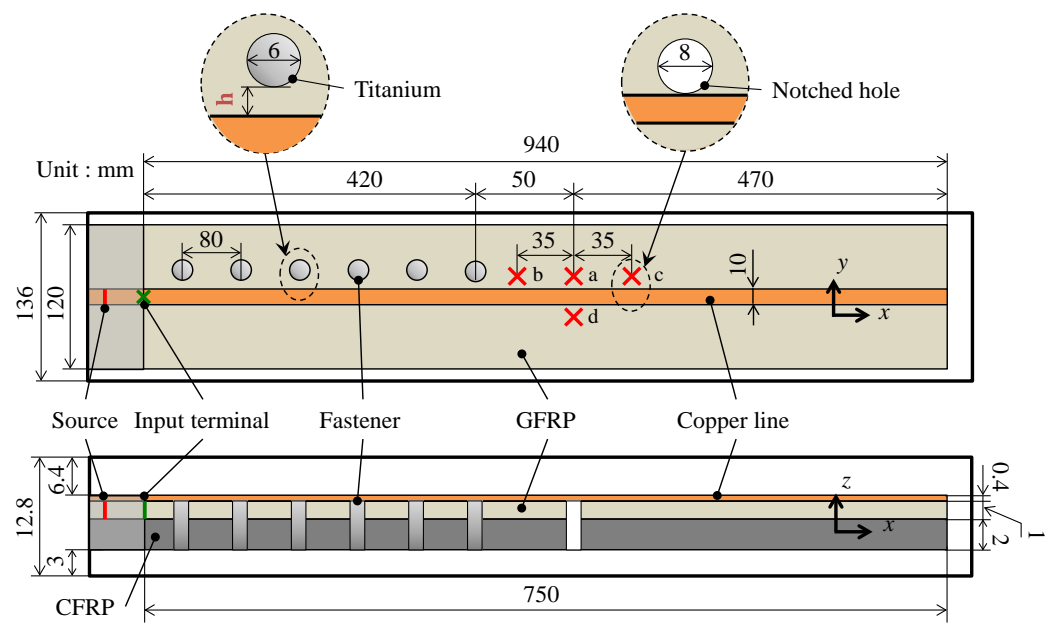

Figure 2. Specimen configuration for FDTD analysis with fasteners.

where $c$ is the speed of light. To satisfy Courant's stability condition, the time step is set to $\Delta t=0.5 \mathrm{ps}$. The specific magnetic permeability of the entire region is set to $\mu_{r}=1.0$. The specific dielectric constant of air is $\varepsilon_{r}=1.0$, and the specific dielectric constant of GFRP is set to $\varepsilon_{r}=4.0$. The conductivity of copper is $5.96 \times 10^{7} \mathrm{~S} / \mathrm{m}$, and the conductivity of CFRP measured by Hirano et al. [16] was used in this study. A pulse signal was input from the left terminal. Figure 3 shows the input Gaussian pulse signal for the FDTD analysis.

Six fasteners, $6 \mathrm{~mm}$ in diameter, were fabricated on the model with $80-\mathrm{mm}$ spacing. The distance from the MSL is defined as $h$ as shown in Figure 2: $h$ is the distance from the edge of the metallic fastener to the edge of the width of the MSL copper tape. In Figure 2, a typical model of the damage at an open hole is made at one of the cross marks shown as from (a) to (d). The reflected pulse wave from the hole is observed in the analysis. The diameter of the open hole is $8 \mathrm{~mm}$ and the edge of the hole has contact with the MSL copper tape. The effect of distance $\mathrm{h}$ on the reflected signal from the hole is analyzed in this study. Five distances between the fasteners and the MSL $(h)$ are used in the calculations: 1, 2, 3, 4 and $5 \mathrm{~mm}$. The reflected signal from the hole near the MSL is compared for each result. As a reference, the case without a model-damage hole is calculated.

\section{Analytical Results and Discussion}

Figure 4 shows the results of the reflected signal calculated at the input terminal. The abscissa shows the time and the ordinate shows the voltage. The broken curve represents the reflected signal of the specimen without fasteners. As shown in Figure 4, when the fasteners approach the MSL, the amplitudes of the reflected signals decrease. For example, the nearest case of $h=1 \mathrm{~mm}$ had only $11 \%$ of the amplitude compared with the amplitude of the reflected signal without fasteners. The decrease in signal amplitude was caused by the scatter of electromagnetic wave at the metallic fasteners. When $h=4 \mathrm{~mm}$, the amplitude of the reflected signal was $92 \%$. The results showed that the $h=4 \mathrm{~mm}$ was the minimum requirement for the distance between the fasteners and the MSL with these dimensions.

\section{Experimental Method}

Figure 5 shows the dimensions and configuration of the specimen. The specimen was $1750 \mathrm{~mm}$ long, $200 \mathrm{~mm}$ wide and $2.6 \mathrm{~mm}$ deep. The material used to fabricate the CFRP laminate was Toray T800S/epoxy prepreg P2352W-19 (cure temperature $180^{\circ} \mathrm{C}$ ). The stacking sequence of the CFRP plate was [0/45/0/ - 45/90/45/0/ 45/90] . On the CFRP specimen, a GFRP fabric plate $1730 \mathrm{~mm}$ long, $200 \mathrm{~mm}$ wide and $0.5 \mathrm{~mm}$ deep was attached using commercially available epoxy adhesive. On the GFRP plate, copper tape $5 \mathrm{~mm}$ wide and $0.025 \mathrm{~mm}$ thick was attached as shown in Figure 5 to create two micro-strip lines. The triangle symbols indicate the location of fasteners of $6.35 \mathrm{~mm}$ diameter. The spacing of the fasteners was $143.2 \mathrm{~mm}$ for the specimen. The characteristic impedance calculated from the Equations (1) to (4) was approximately $15 \Omega$. Because it was quite difficult to obtain a transmission line of exactly $50 \Omega$ using commercially available GFRP plate and copper tape, the characteristic impedance of the MSL was set to $15 \Omega$ in this study. 


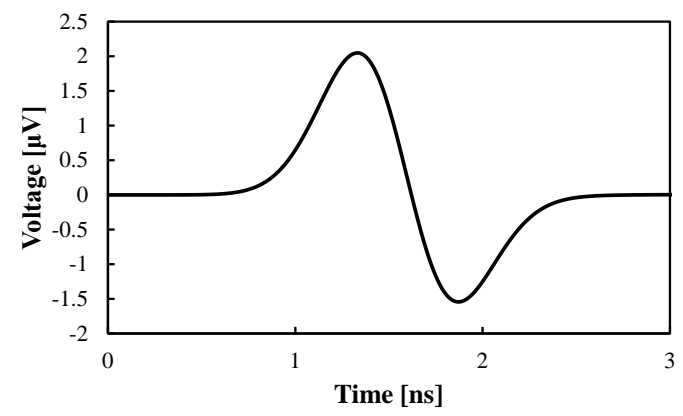

Figure 3. Input signal used for FDTD analysis.

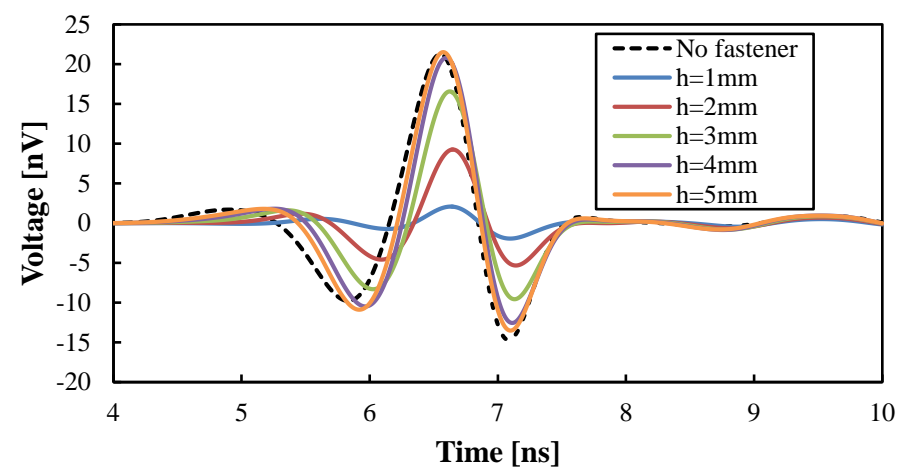

Figure 4. Reflected signal of FDTD analysis: the effect of distance between the fasteners and micro-strip line.

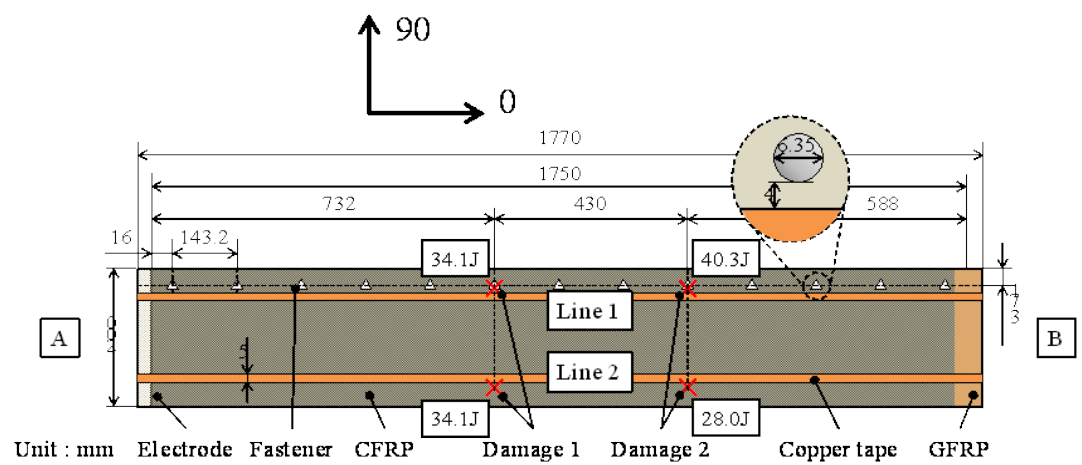

Figure 5. Specimen configurations for the experimental investigation on the effect of fasteners on the self-sensing TDR.

Figure 6 shows a view of the input terminal at the specimen end. A coaxial cable was connected to the microstrip line by soldering as shown in Figure 6. At the CFRP specimen edge, electrical copper plating was carried out to make electrical contact.

Two micro-strip lines were created on the specimen. Line \#1 was placed close to the fasteners (the distance between the fasteners and Line 1 was $4 \mathrm{~mm}$ ). Line \#2 was placed far from the fasteners to compare the results. The left terminal was named "terminal A" and the right terminal "terminal B" in this study. Using both terminals, the relationship between the location of the fastener and the location of the damage was experimentally investigated. For the measurements, the other terminal was set to be open-ended. To prevent specimen warping, $\mathrm{L}$ type aluminum stiffeners $1750 \mathrm{~mm}$ long and $30 \mathrm{~mm}$ wide were attached to the back.

Bearing fracture damage was created at $732 \mathrm{~mm}$ (Damage \#1) and $1162 \mathrm{~mm}$ (Damage \#2) from Terminal A. Bearing failure of a fastener hole in a CFRP plate is usually caused by excessive loading, fatigue loading or initial defects. It is, however, quite difficult to create a bearing failure at a fastener hole edge for this large speci- 


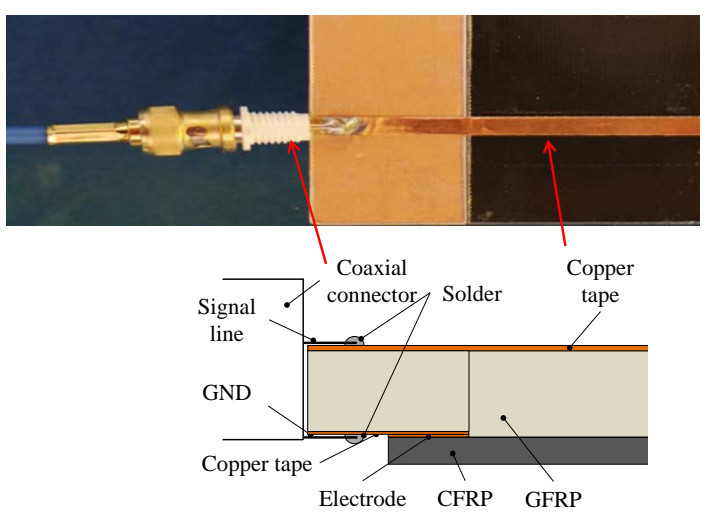

Figure 6. Details of the signal input terminal.

men using a normal material testing machine. In this study, therefore, a drop weight loading was adopted to make a simulated bearing fracture at a fastener as shown in Figure 7. For Line \#1, an impact energy of $34.1 \mathrm{~J}$ was added to Damage \#1, and 40.3 J was added to Damage \#2. For Line \#2, 34.1 J was added to Damage \#1 and 28.0 J was added to Damage \#2. The impact load was applied in the direction of the MSL. The examples of the bearing failures are shown in Figure 8. A white area peeled off the GFRP because of the bearing failure.

Measurements of reflected pulse signals were performed in three conditions as follows. Condition \#0: intact specimen.

Condition \#1: Damage \#1

Condition \#2: Damage \#1 and Damage \#2

Pulse signals were generated using a function generator AFG3251 (Max 240 MHz, Tektronix, Tokyo Japan), and the signal was applied to the MSL. The reflected signals were measured using a digital oscilloscope TDS5034B (sampling interval 4 ps, Tektronix, Tokyo Japan). A directional coupler, ZFDC-10-5 (Mini-Circuit, Yokohama, Japan), was adopted to select the pulse signal wave reflected from the specimen. The pulse signal made by the function generator is shown in Figure 9. The measurements were repeated 100 times, and the averaged value was used as the measured result. A FIR low-pass filter in MATLAB (cut-off frequency is $0.5 \mathrm{GHz}$ ) was used to remove high frequency noise.

\section{Experimental Results and Discussion}

Figure 10 shows the measured results of the reflected signals for Condition $\# 0$. The abscissa is the time and the ordinate is the measured signal voltage. Figure 10 includes the results for Lines \#1 and \#2 of the A and B input terminals. Because the input terminal and the end terminal have slight impedance mismatching, a reflected signal was observed. Using the length of the specimen and the time difference between the input terminal and the end terminal, the average velocity of the pulse signal was calculated. The signal velocity for Line \#1 was 1.474 $\times 10^{8} \mathrm{~m} / \mathrm{s}$ and for Line \#2 was $1.444 \times 10^{8} \mathrm{~m} / \mathrm{s}$. Because the standard deviation of the 100 measurements for Line \#1 was $0.0176 \times 10^{8} \mathrm{~m} / \mathrm{s}$, the difference between Lines \#1 and \#2 was not the result of any measurement error. Line \# 1 had fasteners at a distance of $h=4 \mathrm{~mm}$, and Line \#2 had no fasteners near the MSL. Although the FDTD results in Figure 4 indicated that there was no effect on the fasteners when $h=4$ mm, the experimental results showed that a small increase in signal velocity was obtained when the fasteners were located at the line of $h=4 \mathrm{~mm}$ from the time difference of the peak point in each cases. The measured velocities were used to determine the location of the damage.

The difference in the reflected signals between Condition \#0 and Condition \#1 from Terminal A is shown in Figure 11. Using the tapered cosine window, the reflected signal from the input terminal and the end terminal were eliminated. Because the measured voltage included noise of approximately $3 \mathrm{mV}$, a threshold value of 5 $\mathrm{mV}$ was set to judge the existence of damage. The threshold value can be changed with any other measurement system. Figure 11 indicates that the self-sensing TDR system detected bearing damage at the fastener hole without the effect of fasteners.

Figure 12 shows the difference in the reflected signal between Condition \#0 and Condition \#2 from Terminal A, and indicates that the self-sensing TDR method detects bearing failure at the fastener holes without the effect 


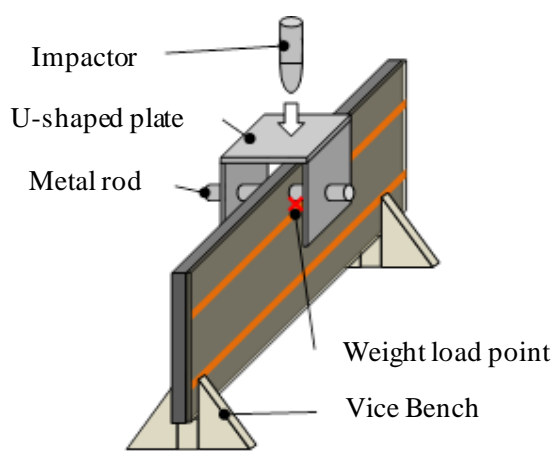

Figure 7. Loading method for creating the bearing failure.

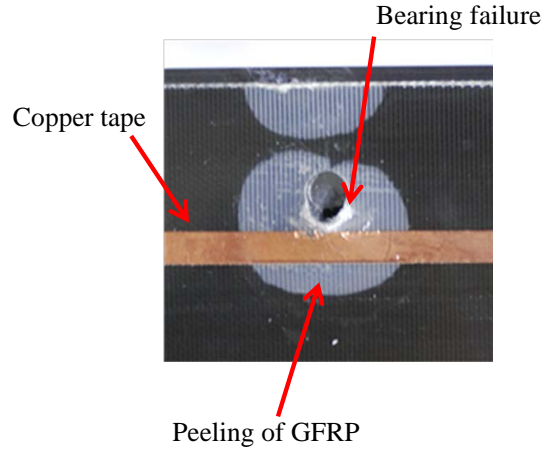

Figure 8. Typical bearing failure (28 J). A fastener was temporally removed to take a photograph.

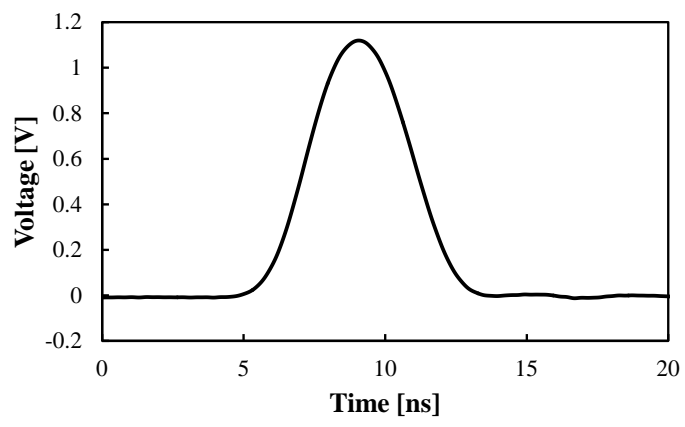

Figure 9. Input pulse signal used for the experiments.

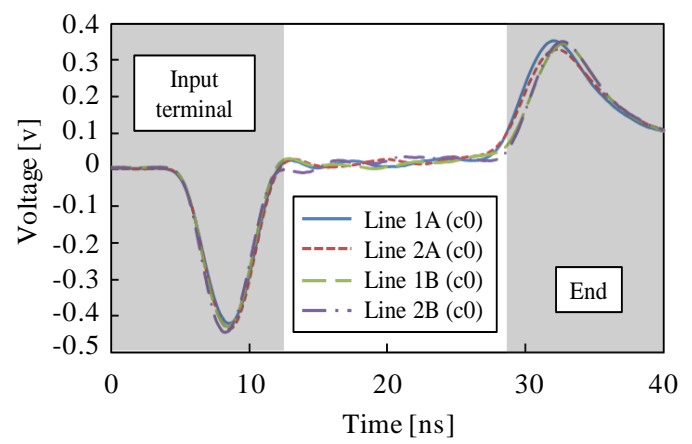

Figure 10. Reflected signals of the specimen without bearing failure damage. 


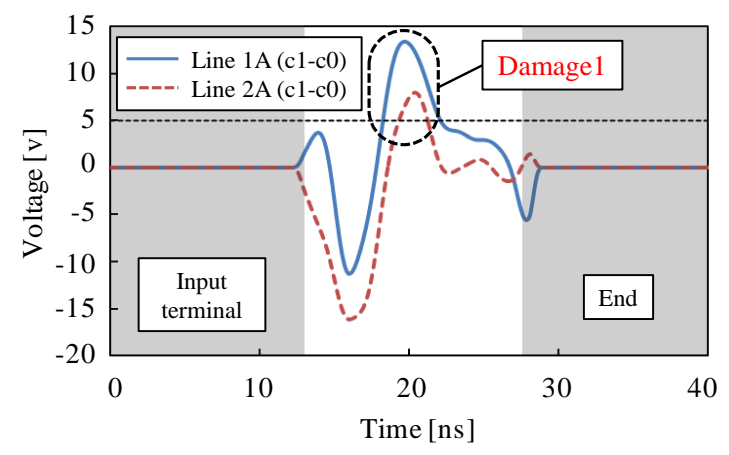

Figure 11. Difference in the reflected signals from Condition $\# 0$ of the results for Condition \#1.

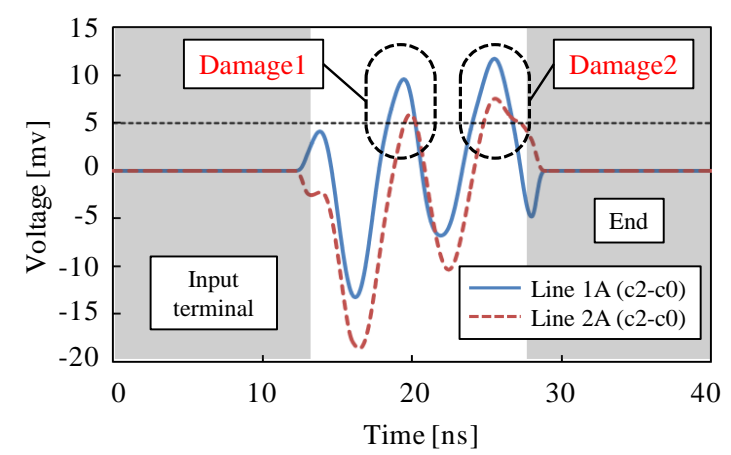

Figure 12. Difference in the reflected signals from Condition \#0 of the results for Condition \#2 from Terminal A.

of fasteners. Figure 13 shows the difference in the reflected signal between Condition \#0 and Condition \#2 from Terminal B. Compared with Figure 12, damage in both cases can be monitored without depending on the order in which it occurred. The results indicated that the self-sensing TDR method was applicable to bearing failure monitoring without considering the MSL.

Using the measured signal velocity and the time difference for the reflected signal, the damage location was identified. Table 1 and Table 2 show the estimation error for the location of damage, which was within $7 \%$ for all cases. These results indicated that the fasteners had no effect on the identification of bearing failure of fastener holes when the distance h (distance from the metallic fastener to the MSL) was set to 4 mm for the selfsensing TDR method.

As shown in Figure 8, some laminate peeled off the GFRP which was caused by the damage created by the bearing failure. The effect of the GFRP peeling will be investigated in future research.

\section{Conclusions}

In this study, a self-sensing TDR was applied to detect the bearing failure of a CFRP laminate with fasteners. Computational design was performed to prevent the impact of electrically conductive fasteners on the measurements of reflected signals. Experiments were conducted to ensure the designed micro-strip line detected the bearing failure of fasteners. The results obtained are as follows:

1) FDTD analysis indicates that the effect of the fasteners can be avoided when the distance from the microstrip line to the fastener is kept to greater than $4 \mathrm{~mm}$.

2) Using the micro-strip line at a distance of $4 \mathrm{~mm}$, the bearing failure of fastener holes can be experimentally detected within $7 \%$ error using the self-sensing TDR.

\section{Acknowledgements}

This research was supported by AOARD-124043. The authors thank Dr. Les Lee of AFOSR for their valuable 


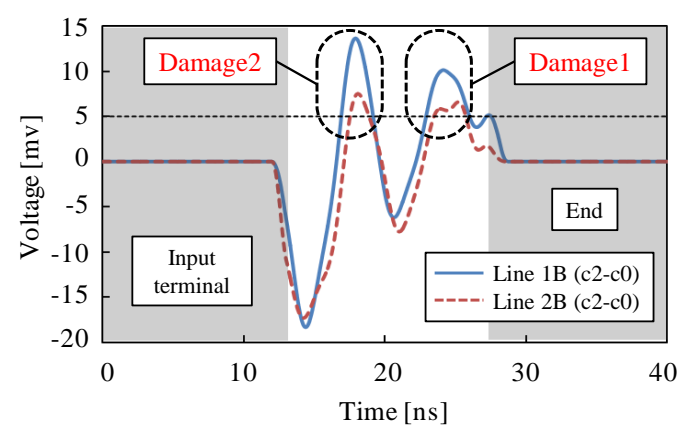

Figure 13. Difference in the reflected signals from Condition \#0 of the results for Condition \#2 from Terminal B.

Table 1. Monitoring results of Condition \#1 (single damage).

\begin{tabular}{ccccc}
\hline & Line\#1A & Line\#1B & Line\#2A & Line\#2B \\
\hline Estimated distance of Damage \#1 [m] & 1.15 & 0.82 & 1.11 & 0.86 \\
Error of location [\%] & 6.89 & 4.60 & 4.78 & 6.73 \\
\hline
\end{tabular}

Table 2. Monitoring results of Condition \#2 (multiple damage).

\begin{tabular}{ccccc}
\hline & Line\#1A & Line\#1B & Line\#2A & Line\#2B \\
\hline Estimated distance of Damage \#1 [m] & 1.15 & 0.80 & 1.12 & 0.82 \\
Estimated distance of Damage \#2 [m] & 0.69 & 1.25 & 0.70 & 1.24 \\
Error of Damage \#1 [\%] & 6.74 & 3.51 & 5.28 & 4.58 \\
Error of Damage \#2 [\%] & 5.11 & 4.55 & 5.75 & 3.62 \\
\hline
\end{tabular}

comments and suggestions. Finally, we would like to thank the AOARD for the grant that made it possible to complete this research. We would also like to thank Dr. Takayuki Nishi of Fuji Heavy Industry Inc. for the partially support of this research.

\section{References}

[1] Thoppul, S.D., Finegan J. and Gibson R.F. (2009) Mechanics of Mechanically Fastened Joints in Polymer-Matrix Composite Structures-A Review. Composites Science and Technology, 69, 301-329. http://dx.doi.org/10.1016/j.compscitech.2008.09.037

[2] Galea, S.C., Chiu, W.K. and Paul, J.J. (1993) Use of Piezoelectric Films in Detecting and Monitoring Damage in Composites. Journal of Intelligent Material Systems and Structures, 4, 330-336. http://dx.doi.org/10.1177/1045389X9300400305

[3] Ihn, J.B. and Chang, F.K. (2004) Detection and Monitoring of Hidden Fatigue Crack Growth Using a Built-In Piezoelectric Sensor/Actuator Network: II. Validation Using Riveted Joints and Repair Patches. Smart Materials and Structures, 13, 621-630. http://dx.doi.org/10.1088/0964-1726/13/3/020

[4] Thostenson, E.T. and Chou, T.W. (2008) Carbon Nanotube-Based Health Monitoring of Mechanically Fastened Composite Joints. Composites Science and Technology, 68, 2557-2561. http://dx.doi.org/10.1016/j.compscitech.2008.05.016

[5] Korokawa, H., Todoroki, A. and Mizutani, Y. (2012) Damage Monitoring of CFRP Plate Using Self-Sensing TDR Method. Journal of Solid Mechanics and Materials Engineering, 6, 1053-1061. http://dx.doi.org/10.1299/jmmp.6.1053

[6] Korokawa, H., Todoroki, A. and Mizutani, Y. (2012) Numerical Simulation of Self-Sensing Time Domain Reflectometry for Damage Detection of Carbon Fiber Reinforced Polymer Plate. Journal of Solid Mechanics and Materials Engineering, 6, 1062-1071. http://dx.doi.org/10.1299/jmmp.6.1062 
[7] Todoroki, A., Kurokawa, H., Mizutani, Y., Matsuzaki, R. and Yasuoka, T. (2014) Self-Sensing Time Domain Reflectometry Method for Damage Monitoring of a CFRP Plate Using a Narrow-Strip Transmission Line. Composites Part B: Engineering, 58, 59-65. http://dx.doi.org/10.1016/j.compositesb.2013.10.047

[8] Todoroki, A., Yamada, K., Mizutani, Y., Suzuki, Y., Matsuzaki, R. and Fujita, H. (2014) Self-Sensing Curved MicroStrip Line Method for Damage Detection of CFRP Composites. Open Journal of Composite Materials, 4, 131-139. http://dx.doi.org/10.4236/ojcm.2014.43015

[9] Matsuzaki, R., Nozawa, Y., Todoroki, A. and Kawasaki, M. (2014) Crack Visualization of Metallic Structures Using Time-Domain Reflectometry with Two-Dimensional Micro Strip Lines. NDT \& E International, 66, 34-42. http://dx.doi.org/10.1016/j.ndteint.2014.04.007

[10] Chen, G.D., Sun, S.S., Pommerenke, D., Drewniak, J.L., Greene, G.G., McDaniel, R.D., Belarbi, A. and Mu, H.M. (2005) Crack Detection of a Full-Scale Reinforced Concrete Girder with a Distributed Cable Sensor. Smart Materials and Structures, 14, S88-S97. http://dx.doi.org/10.1088/0964-1726/14/3/011

[11] Lin, M.W., Thaduri, J. and Abatan, A.O. (2005) Development of an Electrical Time Domain Reflectometry (ETDR) Distributed Strain Sensor. Measurement Science and Technology, 16, 1495-1505. http://dx.doi.org/10.1088/0957-0233/16/7/012

[12] Yankielun, N. and Zabilansky, L. (1999) Laboratory Investigation of Time-Domain Reflectometry System for Monitoring Bridge Scour. Journal of Hydraulic Engineering, 125, 1279-1284. http://dx.doi.org/10.1061/(ASCE)0733-9429(1999)125:12(1279)

[13] Obaid, A.A., Yarlagadda, S., Yoon, M.K., Hager, N.E. and Domszy, R.C. (2006) A Time-Domain Reflectometry Method for Automated Measurement of Crack Propagation in Composites during Mode I DCB Testing. Journal of Composite Materials, 40, 2047-2066. http://dx.doi.org/10.1177/0021998306061309

[14] Mur, G. (1981) Absorbing Boundary Conditions for the Finite-Difference Approximation of the Time-Domain Electromagnetic-Field Equations. IEEE Transactions on Electromagnetic Compatibility, EMC-23, 377-382. http://dx.doi.org/10.1109/TEMC.1981.303970

[15] Wadell, B.C. (1991) Transmission Line Design Handbook. Artech House Inc., Norwood, 298-299.

[16] Hirano, Y., Katsumata, S., Iwahori, Y. and Todoroki, A. (2010) Artificial Lightning Testing on Graphite/Epoxy Composite Laminate. Composites Part A: Applied Science and Manufacturing, 41, 1461-1470. http://dx.doi.org/10.1016/j.compositesa.2010.06.008 